\title{
A revised moment error expression for the AIRGA algorithm
}

\author{
Heike Faßbender and Julius Mayer
}

\begin{abstract}
The fully adaptive rational global Arnoldi method (AIRGA) for the modelorder reduction of second-order multi-input multi-output systems with proportional damping is revisited. The method automatically generates a reduced system approximating the transfer function. It is based on a moment-matching approach. The expansion points are determined iteratively. The reduced order and the number of moments matched per expansion point are determined adaptively using a heuristic based on an error estimation. A revised moment error expression is presented as well as some related findings.
\end{abstract}

\section{Introduction}

A continuous time-invariant second-order multi-input multi-output linear dynamical system is of the form

$$
\begin{aligned}
M \ddot{x}(t) & =-D \dot{x}(t)-K x(t)+F u(t), \\
y(t) & =C_{p} x(t)+C_{v} \dot{x}(t),
\end{aligned}
$$

where $M, D, K \in \mathbb{R}^{n \times n}, F \in \mathbb{R}^{n \times m}, C_{p}, C_{v} \in \mathbb{R}^{q \times n}$ are constant matrices. In (1), $x(t) \in \mathbb{R}^{n}$ is the state, $u(t) \in \mathbb{R}^{m}$ is the input, and $y(t) \in \mathbb{R}^{q}$ is the output. The mass matrix $M$ and the stiffness matrix $K$ need not have any specific property (e.g., symmetry, positive definiteness etc.), but only the special case of proportional damping is

Key Words: Model Order Reduction, Krylov Subspace, Global Arnoldi Algorithm, Moment Matching, Second Order, Proportional Damping.

2010 Mathematics Subject Classification: Primary 65F30; Secondary 70J50.

Received: March, 2017.

Revised: June, 2017.

Accepted: August, 2017. 
considered. That is, the damping matrix is chosen as $D=\alpha M+\beta K$ for some choice of real $\alpha$ and $\beta$.

In many cases, the original system dimension $n$ is too large to allow for an efficient simulation of (1). Therefore, the goal of model reduction is to generate a low dimensional system that has, as best as possible, the same characteristics as the original system, but whose simulation requires significantly less computational effort. The reduced system of (1) is described by

$$
\begin{aligned}
\hat{M} \ddot{x}(t) & =-\hat{D} \dot{\hat{x}}(t)-\hat{K} \hat{x}(t)+\hat{F} u(t), \\
\hat{y}(t) & =\hat{C}_{p} \hat{x}(t)+\hat{C}_{v} \dot{\hat{x}}(t),
\end{aligned}
$$

where $\hat{M}, \hat{K}, \hat{D} \in \mathbb{R}^{r \times r}, \hat{F} \in \mathbb{R}^{r \times m}, \hat{C}_{p}, \hat{C}_{v} \in \mathbb{R}^{q \times r}$ and $r \ll n$. In order to capture the relevant features of the original model, the damping matrix $\hat{D}$ of the reduced order model is required to be $\hat{D}=\alpha \hat{M}+\beta \hat{K}$.

We will revisit the fully adaptive rational global Arnoldi method (AIRGA) for the model-order reduction of second-order multi-input multi-output systems with proportional damping [3]. This method uses a projection based on a moment-matching approach in order to compute the reduced order system. The AIRGA algorithm is recalled in Section 2. It makes use of a heuristic based on an error estimation of the moment error in order to adaptively determine the number of moments to be matched per expansion point. It turned out that the moment error given in [3] is not correct. In order to present our key findings, a revised moment error, the AIRGA algorithm has to be discussed in some detail. In particular, some technicalities from well-known facts are needed. For that matter, we also present some known facts whose proofs in the existing literature seem to be gappy. Our main result, a revised moment error, is given in Section 3. Some concluding remarks are given in Section 4. As all proofs are very technical, they have been moved to Section A.

\section{AIRGA revisited}

In this section we briefly review the AIRGA method [3]. This section is longer than usual because we have to present some well-known facts as we need some technical details of their proofs for further discussion. Moreover, we state some known facts whose proofs in the existing literature seem to be gappy.

\section{Moment-Matching and Projection based Model Order Reduction}

The objective is to generate a reduced order system (2) for which the first moments of the transfer function match those of the original system. The transfer function $H(s)$ of (1) is the linear mapping of the Laplace transform $U(s)$ of the input $u(t)$ to the 
Laplace transform $Y(s)$ of the output $y(t), Y(s)=H(s) U(s)$. It is given by

$$
H(s)=\left(C_{p}+s C_{v}\right)\left(s^{2} M+s D+K\right)^{-1} F=:\left(C_{p}+s C_{v}\right) T(s) .
$$

Here and throughout the paper, $s \in \mathbb{C}$ has to be chosen such that $s^{2} M+s D+K$ is nonsingular. The power series expansion of $T(s)$ around an expansion point $s_{i} \in \mathbb{C}$ is given by (see, e.g., [10])

$$
T(s)=\sum_{k=0}^{\infty} T^{(k)}\left(s_{i}\right)\left(s-s_{i}\right)^{k}
$$

where the $k$-th system moments $T^{(k)}\left(s_{i}\right) \in \mathbb{C}^{n \times m}$ at $s_{i}$ are given by

$$
\begin{aligned}
T^{(0)}\left(s_{i}\right) & =L_{i}^{-1} F \\
T^{(1)}\left(s_{i}\right) & =L_{i}^{-1} B_{i} T^{(0)}\left(s_{i}\right), \quad \text { and for } k=2,3, \ldots \\
T^{(k)}\left(s_{i}\right) & =L_{i}^{-1}\left[B_{i} T^{(k-1)}\left(s_{i}\right)-M T^{(k-2)}\left(s_{i}\right)\right]
\end{aligned}
$$

with

$$
L_{i}=s_{i}^{2} M+s_{i} D+K \quad \text { and } \quad B_{i}=-\left(2 s_{i} M+D\right) .
$$

From (4) we obtain

$$
\begin{aligned}
H(s) & =\sum_{k=0}^{\infty}\left(C_{p}+s C_{v}\right) T^{(k)}\left(s_{i}\right)\left(s-s_{i}\right)^{k} \\
& =\sum_{k=0}^{\infty}\left(C_{p}+s_{i} C_{v}\right) T^{(k)}\left(s_{i}\right)\left(s-s_{i}\right)^{k}+C_{v} T^{(k)}\left(s_{i}\right)\left(s-s_{i}\right)^{k+1} \\
& =: \sum_{k=0}^{\infty} h_{k}\left(s_{i}\right)\left(s-s_{i}\right)^{k}
\end{aligned}
$$

with the moments $h_{0}\left(s_{i}\right)=\left(C_{p}+s_{i} C_{v}\right) T^{(0)}\left(s_{i}\right)$, and for $k=1,2, \ldots$

$$
h_{k}\left(s_{i}\right)=C_{v} T^{(k-1)}\left(s_{i}\right)+\left(C_{p}+s_{i} C_{v}\right) T^{(k)}\left(s_{i}\right) \in \mathbb{C}^{q \times m} .
$$

Similarly, the transfer function of the reduced system (2) is given by

$$
\hat{H}(s)=\left(\hat{C}_{p}+s \hat{C}_{v}\right) \hat{T}(s),
$$

with $\hat{T}(s)=\left(s^{2} \hat{M}+s \hat{D}+\hat{K}\right)^{-1} \hat{F}$. Clearly, here $s \in \mathbb{C}$ has to be chosen such that not only $L=s^{2} M+s D+K$ is nonsingular, but also such that $\hat{L}=s^{2} \hat{M}+s \hat{D}+\hat{K}$ is nonsingular as well. In a projection based framework as considered below this will be satisfied automatically, as $\hat{L}=V^{H} L V$ is nonsingular if $L$ is nonsingular and $V$ is a $n \times r$ matrix with linearly independent columns. 
The power series expansion of $\hat{T}(s)$ around an expansion point $s_{i} \in \mathbb{C}$ is given by

$$
\hat{T}(s)=\sum_{k=0}^{\infty} \hat{T}^{(k)}\left(s_{i}\right)\left(s-s_{i}\right)^{k},
$$

where $\hat{T}^{(k)}\left(s_{i}\right) \in \mathbb{C}^{r \times m}$ is defined analogously to $T^{(k)}\left(s_{i}\right)$. The moments $\hat{h}_{k}\left(s_{i}\right)$ of the reduced system are thus defined analogously to $h_{k}\left(s_{i}\right)$ for $k \in \mathbb{N}_{0}$.

The goal of the moment-matching approach is to find a reduced order model such that the first few moments of (7) match those of (3), that is,

$$
h_{k}\left(s_{i}\right)=\hat{h}_{k}\left(s_{i}\right) \quad \text { for } \quad k=0,1, \ldots, k_{i}-1
$$

for some $k_{i} \in \mathbb{N}$.

A projection based method to generate a reduced oder model of order $r$ constructs a projection $\Pi=V V^{\dagger}$ with a full rank matrix $V \in \mathbb{C}^{n \times r}$ and the pseudoinverse $V^{\dagger}=$ $\left(V^{H} V\right)^{-1} V^{H}$. Since $\Pi=\Pi^{H}$ holds, $\Pi$ is an orthogonal projection. The reduced order model is given by

$$
\begin{aligned}
& V^{\dagger}(M V \ddot{\ddot{x}}(t)+D V \dot{\hat{x}}(t)+K V \hat{x}(t)-F u(t))=0, \\
& \hat{y}(t)=C_{p} V \hat{x}(t)+C_{v} V \dot{\hat{x}}(t) .
\end{aligned}
$$

Thus, we have

$$
\hat{M}=V^{\dagger} M V, \hat{D}=V^{\dagger} D V, \hat{K}=V^{\dagger} K V, \hat{F}=V^{\dagger} F, \hat{C}_{p}=C_{p} V \text { and } \hat{C}_{v}=C_{v} V .
$$

The following well-known theorem $[5,1,9]$ states how to choose $V$ in order to achieve the desired moment-matching property. We restate the theorem as we will need the relation (12) later on.

Theorem 2.1. Assume $s_{i}$ is chosen such that $L_{i}$ is nonsingular. Let $V \in \mathbb{C}^{n \times r}$ have linearly independent columns such that

$$
\operatorname{colspan}(V) \supset \operatorname{colspan}\left(\left[T^{(0)}\left(s_{i}\right), T^{(1)}\left(s_{i}\right), \ldots, T^{\left(k_{i}-1\right)}\left(s_{i}\right)\right]\right) .
$$

Then for the reduced order system (9) it holds that

$$
T^{(k)}\left(s_{i}\right)=V \hat{T}^{(k)}\left(s_{i}\right)
$$

and thus the moment-matching property $h_{k}\left(s_{i}\right)=\hat{h}_{k}\left(s_{i}\right)$ holds for $k=0,1, \ldots, k_{i}-1$.

\section{First and second-order Krylov Subspace}

Theorem 2.1 tells us how to choose $V$. A numerically efficient and stable way to obtain such $V$ makes use of Krylov subspace methods. 
A first-order Krylov subspace $\mathcal{K}_{k}(P, Q)$ of order $k \in \mathbb{N}$ generated by an $n \times n$ matrix $P$ and an $n \times m$ matrix $Q$ is the linear subspace spanned by the columns of the images of $Q$ under powers of $P$

$$
\mathcal{K}_{k}(P, Q)=\operatorname{colspan}\left(\left[Q, P Q, P^{2} Q, \ldots, P^{k-1} Q\right]\right) .
$$

A second-order Krylov subspace $\mathcal{G}_{k}\left(P_{1}, P_{2}, Q\right)$ of order $k$ for $n \times n$ matrices $P_{1}, P_{2}$ and an $n \times m$ matrix $Q$ is defined as follows:

$$
\mathcal{G}_{k}\left(P_{1}, P_{2}, Q\right)=\operatorname{colspan}\left(\left[G_{0}, G_{1}, \ldots, G_{k-1}\right]\right)
$$

with $G_{0}=Q, G_{1}=P_{1} G_{0}$ and $G_{j}=P_{1} G_{j-1}+P_{2} G_{j-2}, j=2,3, \ldots, k-1$.

As already observed in [1], the system moments $T^{(k)}\left(s_{i}\right)$ are just the blocks of the second-order Krylov subspace $\mathcal{G}_{k_{i}}\left(L_{i}^{-1} B_{i}, L_{i}^{-1} M, L_{i}^{-1} F\right)$; that is

$$
\operatorname{colspan}\left(\left[T^{(0)}\left(s_{i}\right), T^{(1)}\left(s_{i}\right), \ldots, T^{\left(k_{i}-1\right)}\left(s_{i}\right)\right]\right)=\mathcal{G}_{k_{i}}\left(L_{i}^{-1} B_{i},-L_{i}^{-1} M, L_{i}^{-1} F\right) .
$$

This also follows directly from (5).

For the special case of proportionally damped second-order systems, the secondorder Krylov subspace is essentially identical to a first order Krylov subspace. This has already been observed, e.g., in [2, 5], but no discussion given so far seems to include all special cases. Let us first consider the following lemma (a similar relation has already been noted in [5, Section 3]); for a proof see the Appendix.

Lemma 2.2. Assume $s_{i}$ is chosen such that $L_{i}$ is nonsingular and $s_{i} \beta \neq-1$. Then

$$
L_{i}^{-1} B_{i}=-\left(\gamma_{i, 1} I_{n}+\gamma_{i, 2} L_{i}^{-1} M\right)
$$

with

$$
\gamma_{i, 1}=\frac{\beta}{s_{i} \beta+1} \quad \text { and } \quad \gamma_{i, 2}=s_{i}+\frac{s_{i}+\alpha}{s_{i} \beta+1} .
$$

With the help of Lemma 2.2 the following theorem can be proven (see the Appendix).

Theorem 2.3. Assume $s_{i}$ is chosen such that $L_{i}$ is nonsingular and $s_{i} \beta \neq-1$. Let $\gamma_{i, 1}, \gamma_{i, 2}$ be defined as in Lemma 2.2. Then, for any $k_{i} \in \mathbb{N}$, it holds

$$
\begin{aligned}
& \mathcal{G}_{k_{i}}\left(L_{i}^{-1} B_{i},-L_{i}^{-1} M, L_{i}^{-1} F\right)=\mathcal{K}_{k_{i}}\left(-L_{i}^{-1} M, L_{i}^{-1} F\right), \quad \text { if } \gamma_{i, 2} \neq 0, \\
& \mathcal{G}_{k_{i}}\left(L_{i}^{-1} B_{i},-L_{i}^{-1} M, L_{i}^{-1} F\right)=\mathcal{K}_{\left\lceil k_{i} / 2\right\rceil}\left(-L_{i}^{-1} M, L_{i}^{-1} F\right), \text { if } \gamma_{i, 2}=0 .
\end{aligned}
$$

Remark 2.4. Assume $s_{i} \beta \neq-1$. Note that $\gamma_{i, 2}=0$ holds iff either $\beta=0$ and $s_{i}=-\frac{\alpha}{2}$ or $\beta \neq 0$ and $s_{i}=-\beta^{-1} \pm \beta^{-1} \sqrt{1-\alpha \beta}$. 
In general, the choice $s_{i} \beta=-1$ is not feasible. Assume for a moment that $s_{i} \beta=$ -1 . This implies $s_{i} \neq 0$ and $\beta \neq 0$. As $L_{i}=s_{i}^{2} M+s_{i} D+K=s_{i}\left(s_{i}+\alpha\right) M$ must be nonsingular, it further implies that $M$ has to be nonsingular and $s_{i} \neq-\alpha$. Moreover,

$$
\begin{aligned}
\mathcal{K}_{k_{i}}\left(-L_{i}^{-1} M, L_{i}^{-1} F\right) & =\mathcal{K}_{k_{i}}\left(\left(s_{i}^{2}+\alpha s_{i}\right)^{-1} M^{-1} M, L_{i}^{-1} F\right)=\mathcal{K}_{k_{i}}\left(I_{n}, L_{i}^{-1} F\right) \\
& =\operatorname{colspan}\left(M^{-1} F\right)
\end{aligned}
$$

and by an easy manipulation

$$
\mathcal{G}_{k_{i}}\left(L_{i}^{-1} B_{i},-L_{i}^{-1} M, L_{i}^{-1} F\right)=\mathcal{K}_{k_{i}}\left(M^{-1} K, M^{-1} F\right) .
$$

Therefore, unless $M=\mu K, \mu \in \mathbb{R}$ or $K=0_{n \times n}$, it follows for $s_{i} \beta=-1$

$$
\mathcal{K}_{k_{i}}\left(-L_{i}^{-1} M, L_{i}^{-1} F\right) \not \supset \mathcal{G}_{k_{i}}\left(L_{i}^{-1} B_{i},-L_{i}^{-1} M, L_{i}^{-1} F\right) .
$$

So, when an expansion point $s_{i}$ is chosen, it always has to be checked that $s_{i} \beta \neq-1$ as we will make use of Theorem 2.3 when constructing the matrix $V$.

\section{The Global Arnoldi Method}

Theorem 2.3 suggests to generate the desired matrix $V$ from $\mathcal{K}_{k_{i}}\left(-L_{i}^{-1} M, L_{i}^{-1} F\right)$; in case $\gamma_{i, 2}=0$, only $\mathcal{K}_{\left\lceil k_{i} / 2\right\rceil}\left(-L_{i}^{-1} M, L_{i}^{-1} F\right)$ has to be considered. Standard efficient and numerically sound methods to compute a basis (and thus $V$ ) of a Krylov subspace are, e.g., the block or the global Arnoldi algorithm [7, 6, 8].

The AIRGA method uses the global Arnoldi method. It constructs a basis $W_{i, 1}$, $W_{i, 2}, \ldots, W_{i, k_{i}} \in \mathbb{C}^{n \times m}$ of the Krylov subspace $\mathcal{K}_{k_{i}}\left(P_{i}, Q_{i}\right)$ with $P_{i}=-L_{i}^{-1} M \in \mathbb{C}^{n \times n}$ and $Q_{i}=L_{i}^{-1} F \in \mathbb{C}^{n \times m}$ which is block-orthonormal in the following sense

$$
\begin{array}{ll}
\left\langle W_{i, j}, W_{i, p}\right\rangle=0 & j \neq p, \\
\left\langle W_{i, j}, W_{i, p}\right\rangle=1 & j=p
\end{array} \quad \text { for } j, p=1, \ldots, k_{i} .
$$

Here, $\langle Y, Z\rangle=\operatorname{trace}\left(Y^{H} Z\right)$ where $Y, Z \in \mathbb{C}^{n \times s}$. The associated norm is the Frobenius norm $\|\cdot\|_{F}$.

In order to simplify the discussion, we assume that $k_{i}$ is chosen such that the global Arnoldi algorithm does not break down; that is, for each $s_{i}$ it produces a matrix $W_{i}=\left[\begin{array}{lll}W_{i, 1} & \cdots & W_{i, k_{i}}\end{array}\right] \in \mathbb{C}^{n \times k_{i} \cdot m}$, representing a block-orthonormal basis of the block Krylov subspace $\mathcal{K}_{k_{i}}\left(-L_{i}^{-1} M, L_{i}^{-1} F\right)$. Then the following relation holds for the block-orthonormal matrix $W_{i}$

$$
P_{i} W_{i}=W_{i}\left(H_{k_{i}}^{(i)} \otimes I_{m}\right)+h_{k_{i}+1, k_{i}}^{(i)}\left[0, \ldots, 0, W_{i, k_{i}+1}\right]
$$

with

$$
W_{i, 1}=Q_{i} / h_{1,0}^{(i)}, \quad h_{1,0}^{(i)}=\left\|Q_{i}\right\|_{F} .
$$

Here $H_{k_{i}}^{(i)}$ is an unreduced $k_{i} \times k_{i}$ upper Hessenberg matrix and $\otimes$ denotes the usual Kronecker product. If $m=1$, the global Arnoldi algorithm reduces to the standard Arnoldi algorithm. 


\section{Multiple expansion points}

In order to ensure a good reduced model in the entire problem dependent frequency domain of interest, one usually employs not just one expansion point, but a set of $\ell$ expansion points. That is, one considers a set $S=\left\{s_{1}, \ldots, s_{\ell}\right\}$ of $\ell$ expansion points and the corresponding block Krylov subspaces

$$
\mathcal{K}_{k_{i}}\left(P_{i}, Q_{i}\right)=\mathcal{K}_{k_{i}}\left(-L_{i}^{-1} M, L_{i}^{-1} F\right) \quad \text { for } \quad i=1, \ldots, \ell
$$

together with the associated block-orthonormal basis $W_{i} \in \mathbb{C}^{n \times k_{i} \cdot m}$ (computed by the global Arnoldi algorithm such that each $W_{i}$ satisfies (14).). Recall that the expansion points $s_{i}, i=1, \ldots, \ell$, have to be chosen such that $L_{i}=s_{i}^{2} M+s_{i} D+K$ is nonsingular and $s_{i} \beta \neq-1$.

\section{Generating the projection $\Pi$}

Let

$$
W=\left[\begin{array}{llll}
W_{1} & W_{2} & \ldots & W_{\ell}
\end{array}\right] \in \mathbb{C}^{n \times r_{\max }}, \quad r_{\max }=m \sum_{i=1}^{\ell} k_{i} .
$$

Clearly,

$$
\operatorname{colspan}(W) \supset \operatorname{colspan}\left(W_{i}\right) \supset \operatorname{colspan}\left(T^{(k)}\left(s_{i}\right)\right)
$$

for $i=1,2, \ldots, \ell$ and $k=0,1, \ldots, k_{i}-1$. Now $\Pi=V V^{\dagger}$ can be set up using any full rank matrix $V \in \mathbb{C}^{n \times r}$ which has the same column space as $W$. Then, due to Theorem 2.1 , the first $k_{i}$ moments at the expansion point $s_{i}, i=1, \ldots, \ell$ of the reduced order system (9) generated with $V$ are matching those of the original system (1), that is,

$$
h_{j}\left(s_{i}\right)=\hat{h}_{j}\left(s_{i}\right) \text { holds for } j=0, \ldots, k_{i}-1 \text { and } i=1,2, \ldots, \ell .
$$

\section{Choosing the expansion points iteratively}

Given the number $\ell$ of expansion points, the set $S=\left\{s_{1}, \ldots s_{\ell}\right\}$ of expansion points and the number $k_{i}$ of moments to be matched at each $s_{i}$, the algorithm sketched so far will compute the desired reduced order model. As it is a priori not obvious how to choose $k_{i}$, the AIRGA algorithm [3] chooses the $k_{i}$ adaptively given a fixed set $S$ and the total number of number of moments to be matched, $r_{\max } / \mathrm{m}$. Thus, unlike as described so far, the algorithm does not generate $W_{i}$ corresponding to $\mathcal{K}_{k_{i}}\left(L_{i}^{-1} M, L_{i}^{-1} F\right)$ at once. Instead, the following approach is used: The expansion points are picked iteratively. The first time $s_{i}$ is picked, just $\mathcal{K}_{1}\left(L_{i}^{-1} M, L_{i}^{-1} F\right)$ is used to generate $W_{i} \in \mathbb{C}^{n \times m}$ and just one moment is matched at $s_{i}$. The next time $s_{i}$ is picked, this is expanded to $\mathcal{K}_{2}\left(L_{i}^{-1} M, L_{i}^{-1} F\right)$ and $W_{i} \in \mathbb{C}^{n \times 2 m}$ matching two moments at $s_{i}$, and so forth. Assume that the algorithm has picked the expansions points such that the first $k_{i}$ moments are matched at expansion point $s_{i}$, that is, $h_{k}\left(s_{i}\right)=\hat{h}_{k}\left(s_{i}\right)$ holds for 
$k=0,1, \ldots, k_{i}-1$. The choice of the next expansion point to be considered is based on the $k_{i}$-th moment error at expansion point $s_{i}$

$$
\left\|h_{k_{i}}\left(s_{i}\right)-\hat{h}_{k_{i}}\left(s_{i}\right)\right\|_{F}=\varepsilon_{k_{i}}\left(s_{i}\right) .
$$

The expansion point $s_{p}$ chosen next is the one corresponding to the maximum moment error by $s_{p}=\operatorname{argmax}_{s_{i}} \varepsilon_{k_{i}}\left(s_{i}\right)$.

\section{AIRGA revised}

The idea for the adaptive choice of the expansion points is based on an expression which describes the $k_{i}$-th moment error $\varepsilon_{k_{i}}\left(s_{i}\right)$. The expression given in [3] is not correct. Here is the revised version of the result.

Theorem 3.1. Assume that $s_{i}$ is chosen for all $i=1, \ldots, \ell$, such that $L_{i}=s_{i}^{2} M+$ $s_{i} D+K$ is nonsingular and $s_{i} \beta \neq-1$. Let $W_{i}, i=1, \ldots, \ell$, be computed by the global Arnoldi method such that (14) and (15) hold. Let $W=\left[\begin{array}{llll}W_{1} & W_{2} & \cdots & W_{\ell}\end{array}\right] \in \mathbb{C}^{n \times r_{\max }}$ be as in (16). Let $V \in \mathbb{C}^{n \times r}$ be a full rank matrix which has the same column space as $W$. Let the reduced order system (9) be generated via (10). Then the error of the $k_{i}+1$-th moment at $s_{i}$ can be expressed as

$$
\begin{aligned}
\varepsilon_{k_{i}}\left(s_{i}\right) & :=\left\|h_{k_{i}}\left(s_{i}\right)-\hat{h}_{k_{i}}\left(s_{i}\right)\right\|_{F} \\
& =\left|\gamma_{i, 2}^{k_{i}}\right| \cdot\left(\prod_{k=0}^{k_{i}} h_{k+1, k}^{(i)}\right) \cdot\left\|\left(C_{p}+s_{i} C_{v}\right)\left(I_{n}-V\left(V^{\dagger} L_{i} V\right)^{-1} V^{\dagger} L_{i}\right) W_{i, k_{i}+1}\right\|_{F} .
\end{aligned}
$$

In order to be able to prove Theorem 3.1 the following observation which is inspired by [4, Theorem 2] will be useful.

Lemma 3.2. Let $P_{i} \in \mathbb{C}^{n \times n}, Q_{i} \in \mathbb{C}^{n \times m}, \widetilde{H}_{i}=H_{k_{i}}^{(i)} \otimes I_{m}$ and $E_{i}=e_{i} \otimes I_{m}$, where $e_{i} \in \mathbb{R}^{k_{i}}$ denotes the $k_{i}$-th unit vector. Let $W_{i}$ be computed by the global Arnoldi method such that (14) and (15) hold. Then it holds

$$
P_{i}^{k_{i}} Q_{i}=h_{1,0}^{(i)} W_{i} \widetilde{H}_{i}^{k_{i}} E_{1}+\left(\prod_{k=0}^{k_{i}} h_{k+1, k}^{(i)}\right) W_{i, k_{i}+1} .
$$

Theorem 3.1 gives rise to Algorithm 1. It starts with an initial set of expansion points and automatically and adaptively chooses the number of moments to be matched at each expansion point $s_{i}$ based on Theorem 3.1. This is controlled by the inner while loop starting at line 10 where $V$ is computed.

One can use different methods to obtain a full rank matrix $V \in \mathbb{C}^{n \times r}$ which has the same column space as $W \in \mathbb{C}^{n \times r_{\max }}$. A numerically safe way to generate the matrix $V$ from $W$ is the use of the rank-revealing QR-decomposition of $W$. The relevant part 


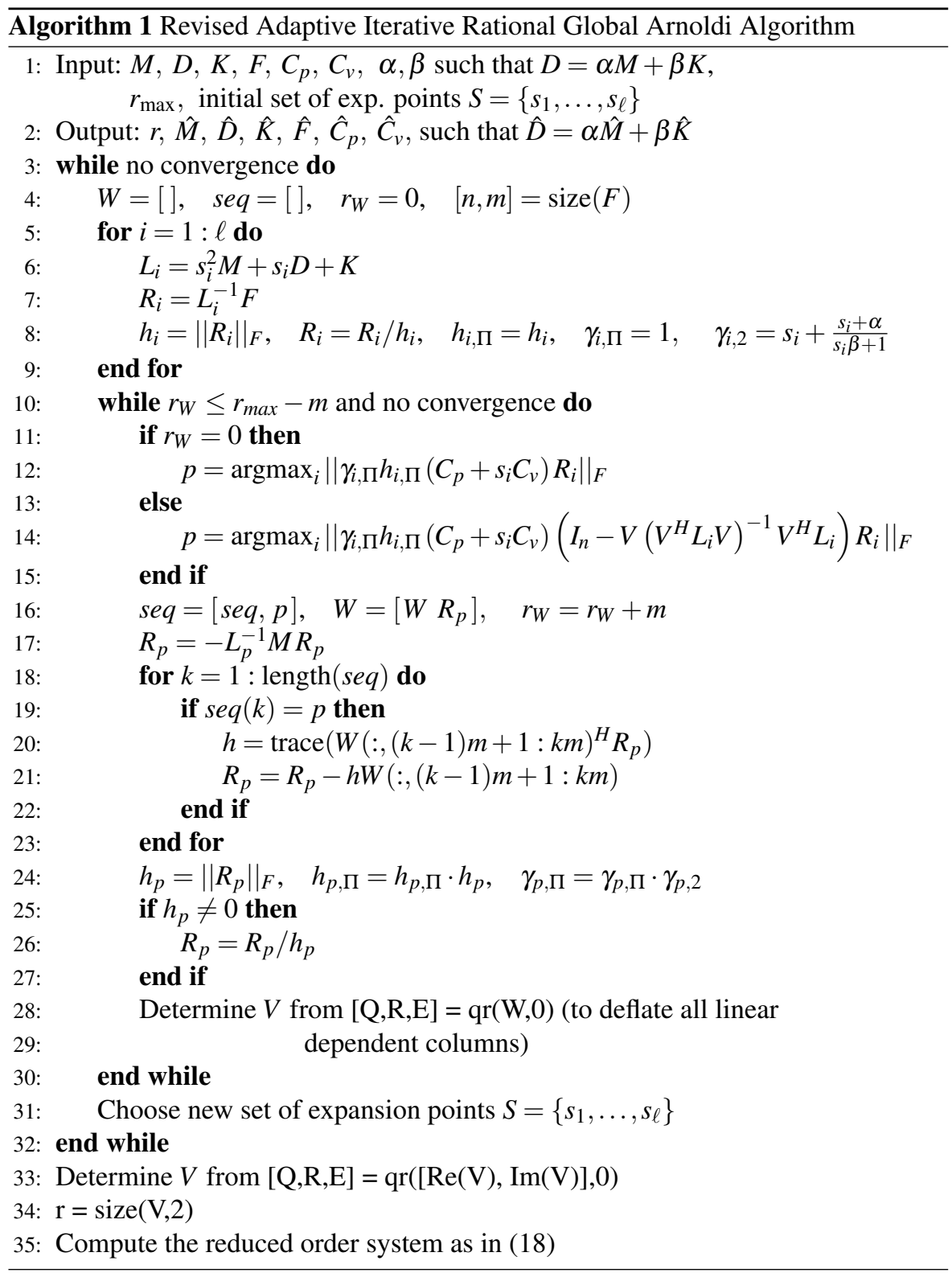


of its unitary factor is then used as $V$ such that $V$ has orthonormal columns. Thus it holds $V^{\dagger}=V^{H}$ and the projection $\Pi=V V^{\dagger}=V V^{H}$ becomes a Galerkin projection. The system matrices of the reduced order system are given by

$$
\hat{M}=V^{H} M V, \hat{D}=V^{H} D V, \hat{K}=V^{H} K V, \hat{F}=V^{H} F, \hat{C}_{p}=C_{p} V \text { and } \hat{C}_{v}=C_{v} V .
$$

The size of the resulting reduced system can not be predetermined. At the end, $V$ will have $r_{\max }$ or less columns.

Please note that $h_{p}$ in line 24 corresponds to a lower subdiagonal element of the associated Hessenberg matrix. In case $h_{p}=0$, we have $R_{p}=0_{n \times m}$. The algorithm does not break down, as this implies that the corresponding moment error is equal to zero. Thus, the corresponding expansion point will not be chosen again.

The quality of the reduced order system heavily depends on the choice of the expansion points. As a good set of expansion points is usually not available, typically such a set is determined iteratively. This is controlled by the outer while loop starting at line 3 of Algorithm 1. One starts with an initial set of expansion points, computes the corresponding reduced order model and selects a new set of expansion points, e.g., based on the eigenvalues of $\lambda^{2} \hat{M}+\lambda \hat{D}+\hat{K}$. The actual selection criterion has to be based on the problem considered, see, e.g., the discussion in [3] and the references therein. This process is repeated till convergence, measured, e.g., in terms of the $\mathrm{H}_{2}$-error between the previously computed reduced order system and the current one

$$
\text { err }=\left\|\hat{H}_{\text {previous }}-\hat{H}_{\text {current }}\right\|_{H_{2}} \text {. }
$$

Here $\|H-G\|_{H_{2}}=\frac{1}{2 \pi} \int_{-\infty}^{\infty}\|H(l \omega)-G(l \omega)\|_{F} d \omega$, where the transfer functions $H$ and $G$ belong to systems with the same input and output dimension. For a thorough discussion on how to determine convergence as well as a new set of expansion points in the outer loop see [3].

Finally note that allowing complex-valued expansion points $s_{i}$ leads to $W \in \mathbb{C}^{n \times r}$. Thus $V$ and the reduced order system (9) is complex-valued, even though usually a real-valued one is desired. Using complex-conjugate pairs of expansion points, at least theoretically, the entire computations can be done in real arithmetic. A different option is to split the complex-valued matrix $V$ into its real and imaginary part and to use a rank-revealing $\mathrm{QR}$ decomposition of $[\operatorname{Re}(V), \operatorname{Im}(V)]$ to obtain a real matrix with orthonormal columns and the same column space. This real-valued matrix may have twice the number of columns as desired. Hence, the dimension of the reduced order system will be doubled. The number of moments matched does not change.

\section{Concluding Remarks}

In [3] the AIRGA algorithm for model-order reduction of second-order multi-input multi-output systems with proportional damping has been proposed. The method 
relies on the moment error $\varepsilon_{k_{i}}\left(s_{i}\right)$ as in (17). Unfortunately the expression for the moment error given in [3] is not correct. Section 3 presents our main contribution: the revised moment error given in Theorem 3.1 as well as Lemma 3.2 which is needed to proof Theorem 3.1. The idea of the AIRGA algorithm and all further details necessary to proof Theorem 3.1 have been summarized in Section 2. In doing so, we have rounded off some of the results employed (by explicitily stating all assumptions and findings which may have been obscured in previous publications). In [3] numerical examples have been considered. Repeating those with the revised moment error do not reveal any major differences in the results. Thus, these experiments have not been included here.

\section{A Proofs}

In this section we provide the details of the proofs for the theorems and lemmas of Sections 2 and 3. For ease of reference, the theorems and lemmas are restated except for Theorem 2.1 which is well-known. Lemma 2.2 and Theorem 2.3 appeared in slightly different form in, e.g., [2, 5], while Lemma 3.2 and Theorem 3.1 are new.

Lemma 2.2 Assume $s_{i}$ is chosen such that $L_{i}$ is nonsingular and $s_{i} \beta \neq-1$. Then

$$
L_{i}^{-1} B_{i}=-\left(\gamma_{i, 1} I_{n}+\gamma_{i, 2} L_{i}^{-1} M\right)
$$

with

$$
\gamma_{i, 1}=\frac{\beta}{s_{i} \beta+1} \quad \text { and } \quad \gamma_{i, 2}=s_{i}+\frac{s_{i}+\alpha}{s_{i} \beta+1}
$$

Proof.

$$
\begin{aligned}
-L_{i}^{-1} B_{i} & =L_{i}^{-1}\left(2 s_{i} M+D\right)=L_{i}^{-1}\left(2 s_{i} M+\alpha M+\beta K\right) \\
& =\left(2 s_{i}+\alpha\right) L_{i}^{-1} M+\beta L_{i}^{-1} K \\
& =\left(2 s_{i}+\alpha\right) L_{i}^{-1} M+\frac{\beta}{s_{i} \beta+1}\left(s_{i} \beta+1\right) L_{i}^{-1} K \\
& =\left(2 s_{i}+\alpha\right) L_{i}^{-1} M+\frac{\beta}{s_{i} \beta+1} L_{i}^{-1}\left(-\left(s_{i}^{2}+s_{i} \alpha\right) M+\left(s_{i}^{2}+s_{i} \alpha\right) M+\left(s_{i} \beta+1\right) K\right) \\
& =\left(2 s_{i}+\alpha-\frac{\beta\left(s_{i}^{2}+s_{i} \alpha\right)}{s_{i} \beta+1}\right) L_{i}^{-1} M+\frac{\beta}{s_{i} \beta+1} L_{i}^{-1}\left(s_{i}^{2} M+s_{i} D+K\right) \\
& =\left(s_{i}+\frac{s_{i}+\alpha}{s_{i} \beta+1}\right) L_{i}^{-1} M+\frac{\beta}{s_{i} \beta+1} L_{i}^{-1} L_{i} \\
& =\gamma_{i, 2} L_{i}^{-1} M+\gamma_{i, 1} I_{n} .
\end{aligned}
$$


Theorem 2.3 Assume $s_{i}$ is chosen such that $L_{i}$ is nonsingular and $s_{i} \beta \neq-1$. Let $\gamma_{i, 1}, \gamma_{i, 2}$ be defined as in Lemma 2.2. Then for any $k_{i} \in \mathbb{N}$ it holds

$$
\begin{aligned}
& \mathcal{G}_{k_{i}}\left(L_{i}^{-1} B_{i},-L_{i}^{-1} M, L_{i}^{-1} F\right)=\mathcal{K}_{k_{i}}\left(-L_{i}^{-1} M, L_{i}^{-1} F\right), \quad \text { if } \gamma_{i, 2} \neq 0, \\
& \mathcal{G}_{k_{i}}\left(L_{i}^{-1} B_{i},-L_{i}^{-1} M, L_{i}^{-1} F\right)=\mathcal{K}_{\left\lceil k_{i} / 2\right\rceil}\left(-L_{i}^{-1} M, L_{i}^{-1} F\right), \text { if } \gamma_{i, 2}=0 .
\end{aligned}
$$

Proof. From $\gamma_{i, 2}=s_{i}+\frac{s_{i}+\alpha}{s_{i} \beta+1}$ it follows that for $\beta \neq 0$

$$
\gamma_{i, 2}=\frac{s_{i}^{2} \beta+2 s_{i}+\alpha}{s_{i} \beta+1}=\frac{s_{i}^{2} \beta^{2}+2 s_{i} \beta+\alpha \beta}{\beta\left(s_{i} \beta+1\right)}=0
$$

iff the numerator $s_{i}^{2} \beta^{2}+2 s_{i} \beta+\alpha \beta$ is zero. This yields $s_{i} \beta=-1 \pm \sqrt{1-\alpha \beta}$.

Assume $\gamma_{i, 2} \neq 0$. Set $P:=-L_{i}^{-1} M, Q:=L_{i}^{-1} F$ such that the blocks of the Krylov subspace $\mathcal{K}_{k}\left(-L_{i}^{-1} M, L_{i}^{-1} F\right)$ are given as $Q, P Q, P^{2} Q, \ldots, P^{k-1} Q$. Since

$$
T^{(0)}\left(s_{i}\right)=L_{i}^{-1} F=Q
$$

we have $\mathcal{G}_{1}\left(L_{i}^{-1} B_{i},-L_{i}^{-1} M, L_{i}^{-1} F\right)=\mathcal{K}_{1}\left(-L_{i}^{-1} M, L_{i}^{-1} F\right)$. Next with Lemma 2.2 , it holds

$$
T^{(1)}\left(s_{i}\right)=L_{i}^{-1} B_{i} L_{i}^{-1} F=-\left(\gamma_{i, 1} I_{n}+\gamma_{i, 2} L_{i}^{-1} M\right) L_{i}^{-1} F=-\gamma_{i, 1} Q-\gamma_{i, 2} P Q
$$

Thus, as $\gamma_{i, 2} \neq 0$ we have $\mathcal{G}_{2}\left(L_{i}^{-1} B_{i},-L_{i}^{-1} M, L_{i}^{-1} F\right)=\mathcal{K}_{2}\left(-L_{i}^{-1} M, L_{i}^{-1} F\right)$.

Now, assume $\mathcal{G}_{j}\left(L_{i}^{-1} B_{i},-L_{i}^{-1} M, L_{i}^{-1} F\right)=\mathcal{K}_{j}\left(-L_{i}^{-1} M, L_{i}^{-1} F\right)$ for $j=1,2, \ldots, p$. Then we can find $\mu_{i}^{(j-1, k)} \in \mathbb{C}$ for $k=0,1, \ldots, j-1$ and $j=1,2, \ldots, p$ such that $T^{(j-1)}\left(s_{i}\right)=\sum_{k=0}^{j-1} \mu_{i}^{(j-1, k)} P^{k} Q$. With Lemma 2.2 it follows

$$
\begin{aligned}
T^{(p)}\left(s_{i}\right) & =L_{i}^{-1} B_{i} T^{(p-1)}\left(s_{i}\right)-L_{i}^{-1} M T^{(p-2)}\left(s_{i}\right) \\
& =-\left(\gamma_{i, 1} I_{n}+\gamma_{i, 2} L_{i}^{-1} M\right) T^{(p-1)}\left(s_{i}\right)-L_{i}^{-1} M T^{(p-2)}\left(s_{i}\right) \\
& =-\gamma_{i, 1} T^{(p-1)}\left(s_{i}\right)-\gamma_{i, 2} P T^{(p-1)}\left(s_{i}\right)-P T^{(p-2)}\left(s_{i}\right) \\
& =-\gamma_{i, 1} \sum_{k=0}^{p-1} \mu_{i}^{(p-1, k)} P^{k} Q-\gamma_{i, 2} P \sum_{k=0}^{p-1} \mu_{i}^{(p-1, k)} P^{k} Q-P \sum_{k=0}^{p-2} \mu_{i}^{(p-2, k)} P^{k} Q \\
& =-\gamma_{i, 1} \sum_{k=0}^{p-1} \mu_{i}^{(p-1, k)} P^{k} Q-\gamma_{i, 2} \sum_{k=1}^{p} \mu_{i}^{(p-1, k-1)} P^{k} Q-\sum_{k=1}^{p-1} \mu_{i}^{(p-2, k-1)} P^{k} Q \\
& =-\gamma_{i, 1} \mu_{i}^{(p-1,0)} Q-\sum_{k=1}^{p-1}\left(\gamma_{i, 1} \mu_{i}^{(p-1, k)}+\gamma_{i, 2} \mu_{i}^{(p-1, k-1)}+\mu_{i}^{(p-2, k-1)}\right) P^{k} Q
\end{aligned}
$$




$$
\begin{aligned}
& -\gamma_{i, 2} \mu_{i}^{(p-1, p-1)} P^{p} Q \\
= & : \sum_{k=0}^{p} \mu_{i}^{(p, k)} P^{k} Q .
\end{aligned}
$$

The above directly reveals the recursion formula

$$
\begin{aligned}
& \mu_{i}^{(p, 0)}=-\gamma_{i, 1} \mu_{i}^{(p-1,0)} \\
& \mu_{i}^{(p, k)}=-\gamma_{i, 1} \mu_{i}^{(p-1, k)}-\gamma_{i, 2} \mu_{i}^{(p-1, k-1)}-\mu_{i}^{(z-2, k-1)}, \quad \text { for } k=1,2, \ldots, p-1 \\
& \mu_{i}^{(p, p)}=-\gamma_{i, 2} \mu_{i}^{(p-1, p-1)}
\end{aligned}
$$

for any $p \geq 2$ with $\mu_{i}^{(0,0)}=1, \mu_{i}^{(1,0)}=-\gamma_{i, 1}$ and $\mu_{i}^{(1,1)}=-\gamma_{i, 2}$. Particularly, it holds

$$
\mu_{i}^{(p, p)}=\left(-\gamma_{i, 2}\right)^{p}
$$

As $\gamma_{i, 2} \neq 0$, we immediately have $\mathcal{G}_{k}\left(L_{i}^{-1} B_{i},-L_{i}^{-1} M, L_{i}^{-1} F\right)=\mathcal{K}_{k}\left(-L_{i}^{-1} M, L_{i}^{-1} F\right)$, so that the first equation of the theorem is proven by induction.

In order to prove the second statement of the theorem, assume $\gamma_{i, 2}=0$. With Lemma 2.2, it follows

$\mathcal{G}_{k_{i}}\left(L_{i}^{-1} B_{i},-L_{i}^{-1} M, L_{i}^{-1} F\right)=\mathcal{G}_{k_{i}}\left(-\gamma_{i, 1} I_{n},-L_{i}^{-1} M, L_{i}^{-1} F\right)=\mathcal{K}_{\left\lceil k_{i} / 2\right\rceil}\left(-L_{i}^{-1} M, L_{i}^{-1} F\right)$.

Lemma 3.2 Let $P_{i} \in \mathbb{C}^{n \times n}, Q_{i} \in \mathbb{C}^{n \times m}, \widetilde{H}_{i}=H_{k_{i}}^{(i)} \otimes I_{m}$ and $E_{i}=e_{i} \otimes I_{m}$, where $e_{i} \in \mathbb{R}^{k_{i}}$ denotes the $k_{i}$-th unit vector. Let $W_{i}$ be computed by the global Arnoldi method such that (14) and (15) hold. Then it holds

$$
P_{i}^{k_{i}} Q_{i}=h_{1,0}^{(i)} W_{i} \widetilde{H}_{i}^{k_{i}} E_{1}+\left(\prod_{k=0}^{k_{i}} h_{k+1, k}^{(i)}\right) W_{i, k_{i}+1} .
$$

\section{Proof. Observe}

$$
P_{i} W_{i}=W_{i}\left(H_{k_{i}}^{(i)} \otimes I_{m}\right)+h_{k_{i}+1, k_{i}}^{(i)}\left[0, \ldots, 0, W_{i, k_{i}+1}\right]=W_{i} \tilde{H}_{i}+h_{k_{i}+1, k_{i}}^{(i)} W_{i, k_{i}+1} E_{k_{i}}^{T} .
$$

Multiplication from the left by $P_{i}^{k_{i}-1}$ and repeated use of (21) yields

$$
\begin{aligned}
P_{i}^{k_{i}} W_{i} & =P_{i}^{k_{i}-2}\left(P_{i} W_{i}\right) \widetilde{H}_{i}+P_{i}^{k_{i}-1} h_{k_{i}+1, k_{i}}^{(i)} W_{i, k_{i}+1} E_{k_{i}}^{T} \\
& =P_{i}^{k_{i}-2}\left(W_{i} \widetilde{H}_{i}+h_{k_{i}+1, k_{i}}^{(i)} W_{i, k_{i}+1} E_{k_{i}}^{T}\right) \widetilde{H}_{i}+h_{k_{i}+1, k_{i}}^{(i)} P_{i}^{k_{i}-1} W_{i, k_{i}+1} E_{k_{i}}^{T}
\end{aligned}
$$




$$
\begin{aligned}
= & P_{i}^{k_{i}-3}\left(P_{i} W_{i}\right) \widetilde{H}_{i}^{2}+h_{k_{i}+1, k_{i}}^{(i)} \sum_{k=0}^{1} P^{k_{i}-1-k} W_{i, k_{i}+1} E_{k_{i}}^{T} \widetilde{H}_{i}^{k} \\
= & P_{i}^{k_{i}-3}\left(W_{i} \widetilde{H}_{i}+h_{k_{i}+1, k_{i}}^{(i)} W_{i, k_{i}+1} E_{k_{i}}^{T}\right) \widetilde{H}_{i}^{2} \\
& \quad+h_{k_{i}+1, k_{i}}^{(i)} \sum_{k=0}^{1} P^{k_{i}-1-k} W_{i, k_{i}+1} E_{k_{i}}^{T} \widetilde{H}_{i}^{k} \\
& \quad P_{i}^{k_{i}-4}\left(P_{i} W_{i}\right) \widetilde{H}_{i}^{3}+h_{k_{i}+1, k_{i}}^{(i)} \sum_{k=0}^{2} P^{k_{i}-1-k} W_{i, k_{i}+1} E_{k_{i}}^{T} \widetilde{H}_{i}^{k} \\
= & \ldots \\
= & W_{i} \widetilde{H}_{i}^{k_{i}}+h_{k_{i}+1, k_{i}}^{(i)} \sum_{k=0}^{k_{i}-1} P^{k_{i}-1-k} W_{i, k_{i}+1} E_{k_{i}}^{T} \widetilde{H}_{i}^{k} .
\end{aligned}
$$

As $Q_{i}=h_{1,0}^{(i)} W_{i, 1}=h_{1,0}^{(i)} W_{i} E_{1}$, we have

$$
P_{i}^{k_{i}} Q_{i}=h_{1,0}^{(i)} P_{i}^{k_{i}} W_{i} E_{1}=h_{1,0}^{(i)} W_{i} \widetilde{H}_{i}^{k_{i}} E_{1}+h_{1,0}^{(i)} h_{k_{i}+1, k_{i}}^{(i)} \sum_{k=0}^{k_{i}-1} P^{k_{i}-1-k} W_{i, k_{i}+1} E_{k_{i}}^{T} \widetilde{H}_{i}^{k} E_{1}
$$

Since $H_{i}$ is an upper Hessenberg matrix, $\widetilde{H}_{i}$ is a block upper Hessenberg matrix. It follows that

$$
\begin{aligned}
E_{k_{i}}^{T} \widetilde{H}_{i}^{p} E_{1} & =0 \quad \text { for } p=0,1, \ldots, k_{i}-2, \quad \text { and } \\
E_{k_{i}}^{T} \widetilde{H}_{i}^{k_{i}-1} E_{1} & =\prod_{k=1}^{k_{i}-1} h_{k+1, k}^{(i)} I_{m} .
\end{aligned}
$$

Substituting this into (22) gives

$$
\begin{aligned}
P_{i}^{k_{i}} Q_{i} & =h_{1,0}^{(i)} W_{i} \widetilde{H}_{i}^{k_{i}} E_{1}+h_{1,0}^{(i)} h_{k_{i}+1, k_{i}}^{(i)} W_{i, k_{i}+1} \prod_{k=1}^{k_{i}-1} h_{k+1, k}^{(i)} I_{m} \\
& =h_{1,0}^{(i)} W_{i} \widetilde{H}_{i}^{k_{i}} E_{1}+\prod_{k=0}^{k_{i}} h_{k+1, k}^{(i)} W_{i, k_{i}+1}
\end{aligned}
$$

Theorem 3.1 Assume that $s_{i}$ is chosen for all $i=1, \ldots, \ell$, such that $L_{i}=s_{i}^{2} M+s_{i} D+K$ is nonsingular and $s_{i} \beta \neq-1$. Let $W_{i}, i=1, \ldots, \ell$, be computed by the global Arnoldi method such that (14) and (15) hold. Let $W=\left[\begin{array}{llll}W_{1} & W_{2} & \cdots & W_{\ell}\end{array}\right] \in \mathbb{C}^{n \times r_{\max }}$ be as in (16). Let $V \in \mathbb{C}^{n \times r}$ be a full rank matrix which has the same column space as $W$. Let 
the reduced order system (9) be generated via (10). Then the error of the $k_{i}+1$-th moment at $s_{i}$ can be expressed as

$$
\begin{aligned}
\varepsilon_{k_{i}}\left(s_{i}\right) & :=\left\|h_{k_{i}}\left(s_{i}\right)-\hat{h}_{k_{i}}\left(s_{i}\right)\right\|_{F} \\
& =\left|\gamma_{i, 2}^{k_{i}}\right| \cdot\left(\prod_{k=0}^{k_{i}} h_{k+1, k}^{(i)}\right) \cdot\left\|\left(C_{p}+s_{i} C_{v}\right)\left(I_{n}-V\left(V^{\dagger} L_{i} V\right)^{-1} V^{\dagger} L_{i}\right) W_{i, k_{i}+1}\right\|_{F} .
\end{aligned}
$$

Proof. First consider $k_{i}=0$. Recall $\hat{T}^{(0)}\left(s_{i}\right)=\hat{L}_{i}^{-1} \hat{F}$.

$$
\begin{aligned}
\varepsilon_{0}\left(s_{i}\right) & =\left\|\left(C_{p}+s_{i} C_{v}\right) T^{(0)}\left(s_{i}\right)-\left(\hat{C}_{p}+s_{i} \hat{C}_{v}\right) \hat{T}^{(0)}\left(s_{i}\right)\right\|_{F} \\
& =\left\|\left(C_{p}+s_{i} C_{v}\right) T^{(0)}\left(s_{i}\right)-\left(C_{p}+s_{i} C_{v}\right) V \hat{T}^{(0)}\right\|_{F} \\
& =\left\|\left(C_{p}+s_{i} C_{v}\right)\left(T^{(0)}\left(s_{i}\right)-V \hat{L}_{i}^{-1} \hat{F}\right)\right\|_{F} \\
& =\left\|\left(C_{p}+s_{i} C_{v}\right)\left(T^{(0)}\left(s_{i}\right)-V\left(V^{\dagger} L_{i} V\right)^{-1}\left(V^{\dagger} F\right)\right)\right\|_{F} \\
& =\left\|\left(C_{p}+s_{i} C_{v}\right)\left(T^{(0)}\left(s_{i}\right)-V\left(V^{\dagger} L_{i} V\right)^{-1} V^{\dagger}\left(L_{i} L_{i}^{-1}\right) F\right)\right\|_{F} \\
& =\left\|\left(C_{p}+s_{i} C_{v}\right)\left(I_{n}-V\left(V^{\dagger} L_{i} V\right)^{-1} V^{\dagger} L_{i}\right) T^{(0)}\left(s_{i}\right)\right\|_{F} .
\end{aligned}
$$

Next consider $k_{i}=1$. Recall $\hat{T}^{(1)}\left(s_{i}\right)=\hat{L}_{i}^{-1} \hat{B}_{i} \hat{T}^{(0)}\left(s_{i}\right)$, and $T^{(0)}\left(s_{i}\right)=V \hat{T}^{(0)}\left(s_{i}\right)$, but in general $T^{(1)}\left(s_{i}\right) \neq V \hat{T}^{(1)}\left(s_{i}\right)$.

$$
\begin{aligned}
\varepsilon_{1}\left(s_{i}\right) & =\left\|\left(C_{p}+s_{i} C_{v}\right) T^{(1)}\left(s_{i}\right)-\left(\hat{C}_{p}+s_{i} \hat{C}_{v}\right) \hat{T}^{(1)}\left(s_{i}\right)\right\|_{F} \\
& =\left\|\left(C_{p}+s_{i} C_{v}\right) T^{(1)}\left(s_{i}\right)-\left(C_{p}+s_{i} C_{v}\right) V \hat{L}_{i}^{-1} \hat{B}_{i} \hat{T}^{(0)}\left(s_{i}\right)\right\|_{F} \\
& =\left\|\left(C_{p}+s_{i} C_{v}\right)\left(T^{(1)}\left(s_{i}\right)-V\left(V^{\dagger} L_{i} V\right)^{-1}\left(V^{\dagger} B_{i} V\right) \hat{T}^{(0)}\left(s_{i}\right)\right)\right\|_{F} \\
& =\left\|\left(C_{p}+s_{i} C_{v}\right)\left(T^{(1)}\left(s_{i}\right)-V\left(V^{\dagger} L_{i} V\right)^{-1}\left(V^{\dagger} B_{i}\right)\left(V \hat{T}^{(0)}\left(s_{i}\right)\right)\right)\right\|_{F} \\
& =\left\|\left(C_{p}+s_{i} C_{v}\right)\left(T^{(1)}\left(s_{i}\right)-V\left(V^{\dagger} L_{i} V\right)^{-1}\left(V^{\dagger} B_{i}\right) T^{(0)}\left(s_{i}\right)\right)\right\|_{F} \\
& =\left\|\left(C_{p}+s_{i} C_{v}\right)\left(T^{(1)}\left(s_{i}\right)-V\left(V^{\dagger} L_{i} V\right)^{-1} V^{\dagger}\left(L_{i} L_{i}^{-1}\right) B_{i} T^{(0)}\left(s_{i}\right)\right)\right\|_{F} \\
& =\left\|\left(C_{p}+s_{i} C_{v}\right)\left(I_{n}-V\left(V^{\dagger} L_{i} V\right)^{-1} V^{\dagger} L_{i}\right) T^{(1)}\left(s_{i}\right)\right\|_{F} .
\end{aligned}
$$

Consider $k_{i}>1$. Recall $\hat{T}^{\left(k_{i}\right)}\left(s_{i}\right)=\hat{L}_{i}^{-1}\left(\hat{B}_{i} \hat{T}^{\left(k_{i}-1\right)}\left(s_{i}\right)-\hat{M} \hat{T}^{\left(k_{i}-2\right)}\left(s_{i}\right)\right)$ and $T^{(j)}\left(s_{i}\right)=$ $V \hat{T}^{(j)}\left(s_{i}\right)$, for $j=1, \ldots, k_{i}-1$, but in general $T^{\left(k_{i}\right)}\left(s_{i}\right) \neq V \hat{T}^{\left(k_{i}\right)}\left(s_{i}\right)$.

$\varepsilon_{k_{i}}\left(s_{i}\right)=\left\|\left(C_{p}+s_{i} C_{v}\right) T^{\left(k_{i}\right)}\left(s_{i}\right)-\left(\hat{C}_{p}+s_{i} \hat{C}_{v}\right) \hat{T}^{\left(k_{i}\right)}\left(s_{i}\right)\right\|_{F}$ 


$$
\begin{aligned}
& =\left\|\left(C_{p}+s_{i} C_{v}\right) T^{\left(k_{i}\right)}\left(s_{i}\right)-\left(C_{p}+s_{i} C_{v}\right) V \hat{L}_{i}^{-1}\left(\hat{B}_{i} \hat{T}^{\left(k_{i}-1\right)}\left(s_{i}\right)-\hat{M} \hat{T}^{\left(k_{i}-2\right)}\left(s_{i}\right)\right)\right\|_{F} \\
& =\left\|\left(C_{p}+s_{i} C_{v}\right)\left\{T^{\left(k_{i}\right)}\left(s_{i}\right)-V\left(V^{\dagger} L_{i} V\right)^{-1} V^{\dagger}\left(B_{i} T^{\left(k_{i}-1\right)}\left(s_{i}\right)-M T^{\left(k_{i}-2\right)}\left(s_{i}\right)\right)\right\}\right\|_{F} \\
& =\left\|\left(C_{p}+s_{i} C_{v}\right)\left(I_{n}-V\left(V^{\dagger} L_{i} V\right)^{-1} V^{\dagger} L_{i}\right) T^{\left(k_{i}\right)}\left(s_{i}\right)\right\|_{F} .
\end{aligned}
$$

Consider now $k_{i} \in \mathbb{N}_{0}$ arbitrarily. With (19) and (23), (24) or (25) we obtain further

$$
\varepsilon_{k_{i}}\left(s_{i}\right)=\left\|\sum_{k=0}^{k_{i}} \mu_{i}^{\left(k_{i}, k\right)}\left(C_{p}+s_{i} C_{v}\right)\left(I_{n}-V\left(V^{\dagger} L_{i} V\right)^{-1} V^{\dagger} L_{i}\right) P_{i}^{k} Q_{i}\right\|_{F} .
$$

As $W$ is built as in (16) and the column space of $V$ and $W$ are the same, we have

$$
\operatorname{colspan}(V) \supset \operatorname{colspan}\left(W_{i}\right) \supset \operatorname{colspan}\left(\left[Q_{i}, P_{i} Q_{i}, P_{i}^{2} Q_{i}, \ldots, P_{i}^{k_{i}-1} Q_{i}\right]\right) .
$$

Thus, we can write $P_{i}^{j} Q_{i}$ as a linear combination of the blocks $W_{i, 1}, \ldots, W_{i, j+1}$ and further as

$$
P_{i}^{j} Q_{i}=V A_{i}^{(j)}, \quad j=0, \ldots, k_{i}-1
$$

for some $A_{i}^{(j)} \in \mathbb{C}^{r \times m}$. Applying this relation and Lemma 3.2 we obtain

$$
\begin{gathered}
\varepsilon_{k_{i}}\left(s_{i}\right)=\|\left(\sum_{k=0}^{k_{i}-1} \mu_{i}^{\left(k_{i}, k\right)}\left(C_{p}+s_{i} C_{v}\right)\left(I_{n}-V\left(V^{\dagger} L_{i} V\right)^{-1} V^{\dagger} L_{i}\right) V A_{i}^{(k)}\right) \\
+\mu_{i}^{\left(k_{i}, k_{i}\right)}\left(C_{p}+s_{i} C_{v}\right)\left(I_{n}-V\left(V^{\dagger} L_{i} V\right)^{-1} V^{\dagger} L_{i}\right) P_{i}^{k_{i}} Q_{i} \|_{F} \\
=\left\|\mu_{i}^{\left(k_{i}, k_{i}\right)}\left(C_{p}+s_{i} C_{v}\right)\left(I_{n}-V\left(V^{\dagger} L_{i} V\right)^{-1} V^{\dagger} L_{i}\right) P_{i}^{k_{i}} Q_{i}\right\|_{F} \\
=\left|\mu_{i}^{\left(k_{i}, k_{i}\right)}\right| \cdot\left\|\left(C_{p}+s_{i} C_{v}\right)\left(I_{n}-V\left(V^{\dagger} L_{i} V\right)^{-1} V^{\dagger} L_{i}\right)\left(h_{1,0}^{(i)} W_{i} \widetilde{H}_{i}^{k_{i}} E_{1}+\prod_{k=0}^{k_{i}} h_{k+1, k}^{(i)} W_{i, k_{i}+1}\right)\right\|_{F} \\
=\left|\mu_{i}^{\left(k_{i}, k_{i}\right)}\right| \cdot \|\left(C_{p}+s_{i} C_{v}\right)\left(I_{n}-V\left(V^{\dagger} L_{i} V\right)^{-1} V^{\dagger} L_{i}\right) W_{i} h_{1,0}^{(i)} \widetilde{H}_{i}^{k_{i}} E_{1} \\
+\left(C_{p}+s_{i} C_{v}\right)\left(I_{n}-V\left(V^{\dagger} L_{i} V\right)^{-1} V^{\dagger} L_{i}\right) \prod_{k=0}^{k_{i}} h_{k+1, k}^{(i)} W_{i, k_{i}+1} \|_{F}
\end{gathered}
$$

Since $\operatorname{colspan}(V) \supset \operatorname{colspan}\left(W_{i}\right)$ we have

$$
W_{i}=V A_{W_{i}}
$$

for some $A_{W_{i}} \in \mathbb{C}^{r \times\left(k_{i} \cdot m\right)}$. Recalling $\mu_{i}^{\left(k_{i}, k_{i}\right)}=\left(-\gamma_{i, 2}\right)^{k_{i}}$ from (20) it holds

$$
\varepsilon_{k_{i}}\left(s_{i}\right)=\left|\gamma_{i, 2}^{k_{i}}\right| \cdot \|\left(C_{p}+s_{i} C_{v}\right)\left(I_{n}-V\left(V^{\dagger} L_{i} V\right)^{-1} V^{\dagger} L_{i}\right) V A_{W_{i}} h_{1,0}^{(i)} \widetilde{H}_{i}^{k_{i}} E_{1}
$$




$$
\begin{array}{r}
+\left(C_{p}+s_{i} C_{v}\right)\left(I_{n}-V\left(V^{\dagger} L_{i} V\right)^{-1} V^{\dagger} L_{i}\right) \prod_{k=0}^{k_{i}} h_{k+1, k}^{(i)} W_{i, k_{i}+1} \|_{F} \\
=\left|\gamma_{i, 2}^{k_{i}}\right| \cdot\left\|\left(C_{p}+s_{i} C_{v}\right)\left(I_{n}-V\left(V^{\dagger} L_{i} V\right)^{-1} V^{\dagger} L_{i}\right) \prod_{k=0}^{k_{i}} h_{k+1, k}^{(i)} W_{i, k_{i}+1}\right\|_{F} \\
=\left|\gamma_{i, 2}^{k_{i}}\right| \prod_{k=0}^{k_{i}} h_{k+1, k}^{(i)} \cdot\left\|\left(C_{p}+s_{i} C_{v}\right)\left(I_{n}-V\left(V^{\dagger} L_{i} V\right)^{-1} V^{\dagger} L_{i}\right) W_{i, k_{i}+1}\right\|_{F}
\end{array}
$$

\section{References}

[1] Z. Bai and Y. Su. Dimension reduction of large-scale second-order dynamical systems via a second-order Arnoldi method. SIAM Journal on Scientific Computing, 26(5):1692-1709, 2005.

[2] C. Beattie and S. Gugercin. Krylov-based model reduction of second-order systems with proportional damping. In Proceedings of the 44th IEEE Conference on Decision and Control, pages 2278-2283, 2005.

[3] T. Bonin, H. Faßbender, A. Soppa, and M. Zaeh. A fully adaptive rational global Arnoldi method for the model-order reduction of second-order MIMO systems with proportional damping. Elsevier Mathematics and Computers in Simulation, 122:1-19, 2016.

[4] C.-C. Chu, M.-H. Lai, and W.-S. Feng. Model-order reductions for MIMO systems using global Krylov subspace methods. Mathematics and Computers in Simulation, 79:1153-1164, 2008.

[5] R. Eid, B. Salimbahrami, B. Lohmann, E.B. Rudny, and J.G. Korvink. Parametric Order Reduction of Proportionally Damped Second Order Systems. Technical Reports on Automatic Control vol. TRAC-1, Technische Universität München, München, Germany, 2006.

[6] K. Jbilou, A. Messaoudi, and H. Sadok. Global FOM and GMRES algorithms for matrix equations. Applied Numerical Mathematics, 31(1):49 - 63, 1999.

[7] Y. Saad. Iterative Methods for Sparse Linear Systems. Society for Industrial and Applied Mathematics, Philadelphia, PA, USA, 2003.

[8] M. Sadkane. Block-Arnoldi and Davidson methods for unsymmetric large eigenvalue problems. Numerische Mathematik, 64(1):195-211, 1993. 
[9] B. Salimbahrami and B. Lohmann. Order reduction of large scale second-order systems using Krylov subspace methods. Linear Algebra and its Applications, 415(2):385 - 405, 2005.

[10] J. M. Wang, C. C. Chu, Q. Yu, and E. S. Kuh. On projection-based algorithms for model-order reduction of interconnects. IEEE Transactions on Circuits and Systems I: Fundamental Theory and Applications, 49(11):1563-1585, 2002.

Heike Faßbender

TU Braunschweig

Institut Computational Mathematics

Universitätsplatz 2

38106 Braunschweig, Germany

Email: h.fassbender@tu-braunschweig.de

Julius Mayer (corresponding author)

TU Braunschweig

Institut Computational Mathematics

Universitätsplatz 2

38106 Braunschweig, Germany

juliusmayer@gmx.net 